\section{ETH}

Eidgenössische Technische Hochschule Zürich Swiss Federal Institute of Technology Zurich
Health Ethics \& Policy Lab

Chaire de bioéthique, ETH Zurich

Institut de médecine sociale et préventive

Université de Berne

Tel: 0445051513

Email: persmed@ethz.ch

\title{
Enquête Suisse
}

\section{Votre point de vue sur la recherche en santé personnalisée}

un sondage d'opinion de l'ETH Zurich, Health Ethics \& Policy Lab et l'Université de Berne, Institut de médecine sociale et préventive

Sondage en ligne:

www.persmed.ethz.ch

Mot de passe: $\mathrm{XYZ}$ 


\section{Introduction}

\author{
Bienvenue à notre enquête sur la recherche en \\ santé personnalisée!
}

Imaginez que vous êtes invité à participer à un projet de recherche en santé personnalisée financé par des fonds publics suisses. Ce projet hypothétique recueille des données sur votre santé.

Les types de données recueillies sont des données personnelles (sexe, âge, poids, etc.), des données sur la santé (antécédents médicaux et dossiers médicaux) et des échantillons biologiques (sang, salive, urine, cheveux ou tissus), qui peuvent fournir des renseignements sur votre ADN. Dans ce qui suit, nous les appellerons données et échantillons.

Les données sont stockées dans une biobanque suisse. II s'agit d'une vaste banque de données gérée par des centres de recherche publics tels que les universités. Les données sont confidentielles et sécurisées.

Cette enquête vous demandera ce que vous pensez du fait de partager vos données personnelles, vos données de santé et vos échantillons biologiques avec une telle biobanque pour la recherche en santé personnalisée. Quelles seraient vos préoccupations ? Quelles seraient vos attentes ?

Pour nous aider à comprendre ce qui est important pour vous, veuillez nous faire part de vos opinions et de votre point de vue sur la mise à disposition de données sur la santé et d'échantillons biologiques pour la recherche. L'enquête n'évalue pas ce que vous savez sur ces sujets. Veuillez répondre à l'enquête honnêtement et au mieux de vos capacités.

\section{Informations supplémentaires :}

Pourquoi des personnes atteintes d'une même maladie réagissent-elles si différemment aux traitements ? Pourquoi un médicament fonctionne-t-il très bien chez une personne et pas chez une autre ? Et pourquoi une personne développe-t-elle une maladie et une autre pas?

La recherche en santé personnalisée veut répondre à ces questions afin de traiter les malades de manière plus précise, personnalisée et efficace à l'avenir. La recherche en santé personnalisée utilise également des renseignements génétiques et autres renseignements personnels sur des personnes en bonne santé pour en apprendre davantage sur les causes de certaines maladies et sur les risques de les développer.

Pour ce faire, il est important que les chercheurs aient accès au plus grand nombre possible de données sur la santé et d'échantillons de différentes personnes (ayant un état de santé différent) dans les biobanques qui sont constituées.

\section{Comment remplir le questionnaire}

- Cochez la case appropriée à côté de la réponse choisie: $\boldsymbol{X}$

- Si vous avez coché la mauvaise case par erreur, remplissez-la complètement avec un stylo à bille, puis cochez la case appropriée.

Merci d'avoir accepté de répondre au sondage ! 


\section{Partie 1: Votre attitude}

Nous aimerions commencer par votre point de vue général sur les questions suivantes:

Q1. Avant aujourd'hui, avez-vous déjà entendu parler de la recherche sur des échantillons biologiques (p. ex. sang, salive, urine, cheveux ou tissus)?
Oui
Non

Q2. Quelle est votre opinion générale à l'égard de la recherche sur des échantillons biologiques humains (comme le sang, la salive, l'urine, les cheveux ou les tissus)?

Très négatif

Plutôt négatif

Indifférent

Plutôt positif

Très positif

Q3. Dans quelle mesure pensez-vous qu'il est important pour vous, votre famille ou la société que vous participiez à la recherche en mettant à disposition vos données de santé (antécédents médicaux et dossiers médicaux) et/ou vos échantillons biologiques (sang, salive, urine, cheveux ou tissus) ?

Veuillez noter sur une échelle de 1 = aucune valeur à 5 = valeur élevée.
$\begin{array}{lllll}1 & 2 & 3 & 4 & 5\end{array}$
Je ne sais pas

1. Pour moi personnellement

2. Pour ma famille

3. Für die Gesellschaft allgemein

Q4. Participeriez-vous à un projet de recherche qui utiliserait vos données sur la santé et/ou vos échantillons biologiques?

Oui (passez à la question 4.1)

Non (passez à la question 5)

Note: Même si vous avez répondu non à cette question, nous vous demandons de répondre à l'ensemble de ce sondage. Nous aimerions connaître votre opinion sur la meilleure façon d'organiser la recherche avec des données sur la santé et des échantillons biologiques en fonction de votre point de vue. 
Q4.1 Quels types de données et d'échantillons seriez-vous prêt à mettre à disposition?

Veuillez en choisir autant que possible.

Questionnaires sur mon état de santé

Dossiers médicaux me concernant (numériques ou sur papier)

Les antécédents médicaux de ma famille

Mon échantillon de sang

Mes échantillons biologiques que je peux prendre moi-même (par ex. salive, cheveux, urine, écouvillon buccal)

$\square$ Mes échantillons biologiques qui doivent être prélevés par le personnel médical (p. ex. tissus tumoraux ou de peau)

Mes données sur les réseaux sociaux

Données dérivées d'applications sur ma santé ou mon mode de vie (p. ex. traceur d'exercice, journal alimentaire, fréquence cardiaque, etc.)

Q4.2 Quelles raisons influencent votre participation à cette recherche?

Veuillez sélectionner jusqu'à 3 plus pertinentes.

$\square$ Pour accroître les connaissances scientifiques

Être au service de la société et des générations futures

Par sens général du devoir

Pour améliorer l'ensemble des soins de santé

Pour obtenir un avantage personnel en apprenant quelque chose sur ma santé

Pour le bien de ma famille (pour connaître les maladies génétiquement transmissibles)

Autre raison (veuillez préciser) :

Je ne sais pas

Q5. Lesquelles des préoccupations suivantes avez-vous?

Veuillez sélectionner jusqu'à 3 plus pertinentes.

Je m'inquiète de la recherche impliquant des informations sur mes gènes

J'ai peur de ce que je pourrais découvrir sur mes propres risques pour la santé

$\square$ Je m'inquiète que la confidentialité de mes données ne soit pas préservée

J'ai m'inquiète que quelqu'un pirate et vole mes données

Je m'inquiète que quelqu'un utilise mes données à des fins discriminatoires à mon encontre ou à l'encontre de ma famille (par exemple, par les assurances maladie ou les services financiers) 
$\square$ Je m'inquiète que mes données et échantillons soient utilisés à des fins commerciales ou de marketing plutôt que de recherche

$\square$ Je refuse que d'autres personnes (comme des entreprises privées ou des chercheurs) profitent financièrement de mes données

$\square$ J'ai peur des aiguilles ou des procédures pour obtenir les échantillons

$\square$ Je ne veux pas faire l'effort nécessaire pour participer

$\square$ Je ne reçois pas d'argent pour cela

$\square$ Je n'ai pas le temps de contribuer

Je me fiche de la recherche en santé

$\square$ Aucune raison particulière

Autre (veuillez préciser):

Q6. Si, hypothétiquement, vous faisiez don de vos données et/ou échantillons à une biobanqueou : quelles informations souhaitez-vous recevoir à l'avance?

Veuillez sélectionner jusqu'à 3 types d'informations les plus importants pour vous.

Les types exacts de recherche qui seront effectués

Les avantages et les risques potentiels du don de mes données et/ou échantillons

$\square$ La manière dont les données et les échantillons sont stockés, telle que rendus anonymes, codés ou stockés avec mon nom

$\square$ Qui a accès à mes données et échantillons

$\square$ Qui bénéficiera de la recherche

$\square$ Qui tirera un profit financier de la recherche

$\square$ Les mesures de sécurité pour préserver la confidentialité et la protection des données et des échantillons

$\square$ Aucune de ces informations ne m'aiderait à décider

$\square$ Autre (veuillez préciser) : 


\section{Partie 2: Gestion des données et des échantillons}

Dans cette partie, nous aimerions savoir comment vous pensez que les données et les échantillons mis à disposition devraient être gérés.

Q7. Les biobanques aimeraient pouvoir utiliser vos données et échantillons dans de multiples projets de recherche, même à l'avenir. À quelle fréquence aimeriez-vous que la biobanque vous demande la permission d'utiliser vos données et échantillons dans le cadre de projets de recherche ? (Sachant que votre décision peut toujours être modifiée ou votre accord retire.)

Ne me demandez qu'une seule fois, lorsque je mets à disposition mes données et/ou échantillons
Redemandez-moi pour chaque nouveau projet
Cela dépendrait du type de projet à l'étude
Je ne sais pas

Q8. Sous quelle forme préféreriez-vous que vos données et échantillons soient conservés?

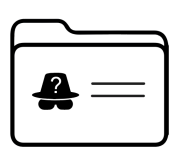

\section{Anonyme}

\section{Explication :}

Les données et les échantillons qui sont anonymisés ne peuvent pas être liés à une personne spécifique sans efforts considérables.

Cela signifie que les identifiants - tels que votre nom, votre adresse, vos photos - sont supprimés de vos données.

\section{Conséquences:}

$\rightarrow$ Vous ne recevrez que les résultats généraux du projet de recherche.

Risque d'entrave à la vie privée:

\section{Faible}

(Les risques pour votre vie privée sont limités, car il est très difficile d'établir un lien entre vos données et vous.)

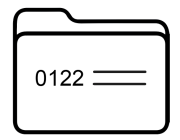

\section{Codée}

\section{Explication:}

Les données et les échantillons sont reliés à une personne spécifique par l'intermédiaire d'un code, lequel est stocké en toute sécurité par les chercheurs. Cela signifie qu'un code ou une clé est nécessaire pour relier les

données à vous.

\section{Conséquences:}

$\rightarrow$ Vous recevez des résultats individuels sur votre statut personnel, en plus des résultats généraux du projet de recherche.

Risque d'entrave à la vie privée: Moyen

(Les risques pour votre vie privée sont limités, car vos données ne peuvent être identifiées qu'à l'aide d'un code sécurisé.)

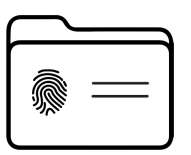

\section{Identifiable}

\section{Explication :}

Les données et les échantillons sont stockés ensemble et étiquetés à votre nom. Ils peuvent facilement être reliés à vous.

\section{Conséquences:}

$\rightarrow$ Vous recevez des résultats individuels sur votre statut personnel, en plus des résultats généraux du projet de recherche.

Risque d'entrave à la vie privée: Élevé

(Votre vie privée peut être menacée, car il est facile d'identifier vos données.) 
Q9. Selon vous, à qui devraient appartenir les données et les échantillons que vous donnez à une biobanque?

Veuillez choisir une seule réponse.

$\square$ A moi personnellement

$\square$ A la biobanque

$\square$ Au gouvernement suisse

$\square$ Aux universités impliquées dans la biobanque

$\square$ Aux chercheurs spécifiques qui font des découvertes

$\square$ A personne

$\square$ Autre (veuillez préciser):

$\square$ Je ne sais pas 


\section{Partie 3: Gouvernance et partage des données}

A présent, nous aimerions savoir comment vous pensez que les données et les échantillons devraient être gérés et partagés.

Q10. Selon vous, qui devrait être responsable de s'assurer que vos données et échantillons sont correctement stockés et gérés?

Veuillez choisir une réponse.

La biobanque (conseil d'administration)

$\square$ Un comité indépendant représentant le public (p. ex. les citoyens, les patients, le public)

Un comité d'experts indépendants ( $p$. ex. chercheurs indépendants : scientifiques et cliniciens non associés à la biobanque)

Un comité mixte de citoyens et d'experts

Autre (veuillez préciser):

Q11. Imaginez que les personnes et organisations suivantes en Suisse aient accès à vos données et échantillons dans la biobanque. Dans quelle mesure auriez-vous confiance qu'ils garderont vos données et échantillons confidentiels et protégés?

Veuillez évaluer sur une échelle allant de 1 = aucune confiance à $5=$ confiance forte.

Mon médecin

Médecins en général

Chercheurs universitaires

Chercheurs d'autres institutions

publics

Entreprises pharmaceutiques

Autres entreprises multi-nationales

Autres entreprises suisses

Assurances maladie

Gouvernement suisse 


\section{Partie 4: Utilité de recevoir des résultats médicaux personnellement pertinents}

Dans cette partie, nous aimerions savoir quels résultats de recherche vous aimeriez recevoir si vous avez mis à disposition des données et des échantillons à une étude.

Q12. Imaginez que vous avez participé à l'étude d'une biobanque et qu'il est possible d'obtenir des résultats de recherche personnels : quels types de résultats de recherche aimeriez-vous recevoir?

Oui Non Je ne sais

1. Renseignements médicaux de base, p. ex. résultats de laboratoire comme les valeurs de sang

2. Comment mon mode de vie influe-t-il sur mon risque de santé (p. ex., tabagisme, poids, etc.)

3. Résultats sur les risques de maladies pour lesquelles il existe des traitements médicales ( $p$. ex. risque de certains types de cancer, certains problèmes cardiaques)

4. Résultats sur les risques de maladies pour lesquelles seules des actions préventives peuvent être entreprises (par exemple, le risque de diabète ou de maladies cardiovasculaires)

5. Résultats sur les risques de maladies pour lesquelles aucun traitement médical n'est disponible, mais qui pourraient avoir une incidence sur mon bien-être ou sur mes décisions de carrière ou planification familiale (p. ex. la maladie d'Alzheimer ou la démence)

6. Résultats de recherche généraux (les résultats ne s'appliquent pas spécifiquement à moi)

Q13. Parfois, les résultats génétiques du développement d'une maladie ne peuvent être exprimés qu'en pourcentage. A quel niveau de probabilité voudriez-vous connaître les résultats ?

Ich möchte Ergebnisse zu meinen Krankheisrisiken erhalten...

Je ne veux recevoir les résultats que si j'aurai définitivement la maladie.

Je veux recevoir les résultats lorsqu'il est très probable que j'attrape la maladie.

Je veux recevoir les résultats quand c'est possible, mais pas très probable que j'attrape la maladie.

Je ne veux pas recevoir de résultats sur les probabilités de maladies.

Je ne sais pas. 
Q14. Si vous voulez recevoir des résultats, par quel canal souhaiteriez-vous les recevoir?

Veuillez cocher toutes les réponses qui s'appliquent.

Lettre par la poste

Appel téléphonique

$\square$ E-Mail

$\square$ Site Web avec connexion sécurisée

$\square$ Application mobile

$\square$ Communication personnelle (face à face)

Q15. Dans quelle mesure l'argent ou toute autre compensation matérielle serait-il important pour vous pour vous motiver à mettre à disposition vos données et/ou échantillons à une biobanque suisse financée par des fonds publics?

Veuillez évaluer sur une échelle de $1=$ pas important à $5=$ très important. 


\section{Dernière partie : Renseignements personnels}

Dans cette dernière partie, nous vous demandons de fournir quelques informations vous concernant.

Q16. Comment décririez-vous votre état de santé général?

$\begin{array}{llll}\text { Très mauvais } & \text { Plutôt mauvais } & \text { Neutre } & \text { Assez bon }\end{array}$

Q17. Quel âge avez-vous?
$18-24$
$\square 35-44$
$55-64$
75 ou plus âgé
$25-34$
65-74

Q18. Was ist Ihr Geschlecht?
$\square$ Homme
Autre
Femme
Préfère ne pas le divulguer

Q19. Avez-vous des enfants biologiques?
Oui
Non

Q20. Quelle est votre nationalité (pays)?

Veuillez cocher toutes les réponses qui s'appliquent.

$\begin{array}{lll}\square \text { Suisse } & \square \text { Allemagne } & \square \text { Kosovo } \\ \square \text { Italie } & \square \text { Portugal } & \square \text { Autre (veuillez préciser): } \\ \square \text { France } & \square \text { Espagne } & \end{array}$

Q21. Quel est votre plus haut niveau d'éducation?

Aucune ou jusqu'à 7 ans d'études obligatoires

École obligatoire (8 ou 9 ans)

Formation professionnelle de base / apprentissage

École secondaire (Maturité)

Formation professionnelle supérieure / apprentissage ou école technique

Diplôme universitaire (Bachelor / Master / Doctorat) 
Q22. Travaillez-vous ou avez-vous déjà travaillé dans le secteur des soins, de la santé, ou de la recherche sur la santé?

$\square$ Oui $\quad \square$ Non

Q23. Vous décririez-vous comme une personne religieuse?
Tout à fait
Un peu
Pas du tout

Remarques générales :

Fin de l'enquête

Merci beaucoup d'avoir pris le temps de répondre à ce sondage!

Nous apprécions grandement votre précieuse contribution!

Veuillez renvoyer le questionnaire complété avant le 23 novembre 2019 à l'adresse suivante. Pour ce faire, vous pouvez utiliser l'enveloppe préaffranchie ci-jointe.

Health Ethics and Policy Lab

ETH Zürich

Hottingerstrasse 10

8092 Zürich 